\title{
The Role of Exercise in Reducing Hyperlipidemia-Induced Neuronal Damage in Apolipoprotein E-Deficient Mice
}

\author{
Yumeng Bai $\mathbb{D}^{1},{ }^{1}$ Yali Feng, ${ }^{1}$ Bo Jiang, ${ }^{2}$ Yan Yang, ${ }^{3}$ Zuowei Pei $\mathbb{D}^{4},{ }^{4}$ Qin Yang $\mathbb{D}^{5}$ \\ and Yanzeng Cui iD ${ }^{1}$ \\ ${ }^{1}$ Department of Neurology, Gongyi People's Hospital, No. 117 Renmin Road, Gongyi 451200, China \\ ${ }^{2}$ Department of Neurological Rehabilitation, Gongyi People's Hospital, No. 117 Renmin Road, Gongyi 451200, China \\ ${ }^{3}$ Department of General Practice, Gongyi People's Hospital, No. 117 Renmin Road, Gongyi 451200, China \\ ${ }^{4}$ Department of Cardiology, Beijing Hospital, National Center of Gerontology, Institute of Geriatric Medicine, Chinese Academy of \\ Medical Sciences, No. 1 Dahua Road, Beijing 100730, China \\ ${ }^{5}$ Department of Internal Medicine, Affiliated Zhongshan Hospital of Dalian University, No. 6 Jiefang Street, Dalian 116001, China
}

Correspondence should be addressed to Yumeng Bai; baiyumeng39600@163.com and Yanzeng Cui; cyz19640371@163.com

Received 27 January 2021; Revised 13 June 2021; Accepted 16 July 2021; Published 9 August 2021

Academic Editor: Vida Demarin

Copyright (C) 2021 Yumeng Bai et al. This is an open access article distributed under the Creative Commons Attribution License, which permits unrestricted use, distribution, and reproduction in any medium, provided the original work is properly cited.

\begin{abstract}
Hyperlipidemia causes nervous system-related diseases. Exercise training has developed into an established evidence-based treatment strategy that is beneficial for neuronal injury. This study investigated the effect of exercise on hyperlipidemia-induced neuronal injury in apolipoprotein E-deficient $\left(\mathrm{ApoE}^{-/-}\right)$mice. Male ApoE $\mathrm{E}^{-/-}$mice (age: 8 weeks) were randomly divided into four groups as follows: mice fed a normal diet (ND), normal diet+swimming training (ND+S), high-fat diet (HD), and high-fat diet+ swimming $(\mathrm{HD}+\mathrm{S})$. Exercise training consisted of swimming for $40 \mathrm{~min} / \mathrm{day}, 5$ days/week for 12 weeks. After 12 weeks, we measured serum levels of total cholesterol (TC), triglyceride (TG), and low-density lipoprotein cholesterol (LDL-c). We also evaluated glial fibrillary acidic protein (GFAP) expression levels using immunohistochemistry, real-time PCR, and immunoblotting. In addition, NLR family pyrin domain-containing 3 (NLRP3), interleukin- (IL-) 18, caspase-1, Bax, Bcl-2, and phosphorylated extracellular signal-regulated kinase (p-ERK) expression levels were measured using immunoblotting. Serum levels of TG, TC, and LDL-c were lower in $\mathrm{ApoE}^{-/-} \mathrm{HD}+\mathrm{S}$ mice than in $\mathrm{ApoE}^{-/-} \mathrm{HD}$ mice. Immunohistochemistry, real-time $\mathrm{PCR}$, and immunoblotting showed increased levels of GFAP in the ApoE ${ }^{-/-} \mathrm{HD}$ group. Immunoblotting revealed increased levels of NLRP3, IL-18, caspase-1, Bax, Bcl-2, and p-ERK in the ApoE ${ }^{-/-}$HD group; however, they were significantly suppressed in the $\mathrm{ApoE}^{-/-} \mathrm{HD}+\mathrm{S}$ group. Therefore, exercise has protective effects against neuronal injury caused by hyperlipidemia.
\end{abstract}

\section{Introduction}

Hyperlipidemia is a lipid metabolism disorder that causes elevated serum total cholesterol (TC), low-density lipoprotein cholesterol (LDL-c), and triglyceride (TG) levels and/or decreased high-density lipoprotein cholesterol levels. Many studies have revealed that hyperlipidemia is a major risk factor for atherosclerosis, cardiocerebrovascular disease, chronic kidney damage, and fatty liver disease $[1,2]$. There is considerable evidence that hyperlipidemia also leads to neuroinflammation, followed by neuronal damage $[3,4]$.
Numerous studies have reported that hyperlipidemia may cause nervous system-related diseases $[5,6]$. For example, hyperlipidemia has been reported to be related to neurodegenerative diseases such as Alzheimer's and Niemann-Pick disease [7, 8]. Paul et al. also reported that hypercholesterolemia increased midbrain dopaminergic neurodegeneration in a mouse model of Parkinson's disease [9]. Hyperlipidemia is an independent risk factor for dementia [10], and high-fat diet- (HD-) induced obesity is associated with an increased risk of type 2 diabetes and impaired neural functions [11]. 
A review article concluded that aerobic exercise and the combination of aerobic and resistance training have effects on the serum levels of cholesterol and lipids [12]. In addition, exercise reduced neuronal injury in several studies. For example, treadmill training significantly increased the formation of lumbar spinal synapses [13]. Similarly, step training after transection of newborn rat spinal cords can increase motor neuron synapse activation [14].

Apolipoprotein E-deficient $\left(\mathrm{ApoE}^{-/-}\right)$mice, a wellestablished animal model of hyperlipidemia, have been used extensively to study the effects of atherosclerosis and renal injury [15-17]. Therefore, we established a hyperlipidemiainduced neuron injury model in $\mathrm{ApoE}^{-/-}$mice by administering a HD and subjecting the mice to exercise in the form of swimming for $40 \mathrm{~min} /$ day, 5 days/week for 12 weeks. This study determined the effect of exercise on hyperlipidemiainduced neuronal injury in $\mathrm{ApoE}^{-/-}$mice and the specific mechanisms involved.

\section{Materials and Methods}

2.1. Animals. Eight-week-old male $\mathrm{ApoE}^{-/-}$mice were obtained from Beijing Vital River Laboratories Animal Technology Co., Ltd. (Beijing, China). All mice were given ad libitum access to food and water and were housed in a room having $40-60 \%$ humidity, at $24-26^{\circ} \mathrm{C}$ with a $12 \mathrm{~h}$ light/dark cycle. $\mathrm{ApoE}^{-/-}$mice were divided randomly into four groups of 7 mice each: normal diet (ND), normal diet+exercise training $(\mathrm{ND}+\mathrm{S})$, high-fat diet (HD), and high-fat diet+swimming exercise training $(\mathrm{HD}+\mathrm{S})$. The $\mathrm{HD}$ mouse food comprised $1.25 \%(w / w)$ cholesterol, $22.5 \%(w / w)$ protein, $20.0 \%(w / w)$ cocoa fat, and $45.0 \%$ carbohydrate (Jiangsu Medicience, Jiangsu, China). One week prior to administration of the test diets, exercise training was initiated in an experimental swimming pool (temperature, $30^{\circ} \mathrm{C}$; water depth, $44 \mathrm{~cm}$; and radius, $120 \mathrm{~cm}$ ). The progressive exercise program initially involved swimming for 5-10 min and was gradually extended to $30 \mathrm{~min} /$ day. When the test diets were implemented, the mice were subjected to formal swimming exercise for $40 \mathrm{~min} /$ day, 5 days/week for 12 weeks. All animal experiments were approved by Gongyi People's Hospital.

2.2. Biochemical Measurements. Blood samples were taken from the abdominal aorta of rats, and serum was stored at $-80^{\circ} \mathrm{C}$. TC, TG, and LDL-c levels were measured using enzyme-linked immunosorbent assay kits (Nanjing Jiancheng Bioengineering Institute, Nanjing, China) according to the manufacturer's protocols. The TC, TG, and LDL-c concentrations were calculated based on measurements of optical density at the respective wavelengths for each compound according to the manufacturer's protocol.

2.3. Immunohistochemistry. The brains of all mice were perfusion-fixed with $4 \%$ paraformaldehyde in $0.1 \mathrm{M}$ sodium phosphate buffer ( $\mathrm{pH}$ 7.4) following a heparinized saline flush. The brains were dehydrated and embedded in paraffin. Serial $7 \mu \mathrm{m}$ coronal sections were cut using a microtome. Paraffin sections of the hippocampus were used for the immunohistochemical analysis, which was performed using the Histone Simple stain kit (Nichirei, Tokyo, Japan) according to the manufacturer's instructions. Paraffin-embedded sections were deparaffinized with xylene and then rehydrated in a descending series of ethanol washes. The sections were treated for $15 \mathrm{~min}$ with $3 \% \mathrm{H}_{2} \mathrm{O}_{2}$ in methanol to inactivate endogenous peroxidases and then incubated at $4^{\circ} \mathrm{C}$ overnight with a primary antibody against glial fibrillary acidic protein (GFAP; rabbit anti-GFAP, 1:500; Z0334, Dako, Carpinteria, CA, USA). All sections were examined microscopically using a BX40 upright light microscope (Olympus, Tokyo, Japan).

2.4. Western Blotting. Mice in each group were euthanized by intraperitoneal injection of an overdose of sodium pentobarbital. The hippocampus and cortex were isolated from each brain ( $n=5$ in each group). Briefly, the hippocampus was homogenized $(1: 5, w: v)$ in ice-cold lysis buffer containing 50 mM Tris- $\mathrm{HCl}$ (pH 7.4), $150 \mathrm{mM} \mathrm{NaCl}, 1 \%$ Nonidet P-40, $1 \mathrm{mM}$ EDTA, $0.25 \%$ sodium deoxycholate, $0.1 \%$ sodium dodecyl sulfate, protease inhibitor cocktail, and phosphatase inhibitor cocktail (1:100 each; Nacalai Tesque, Kyoto, Japan). The resulting homogenates were centrifuged at $12,000 \times g$ for $30 \mathrm{~min}$ at $4^{\circ} \mathrm{C}$, the supernatants were collected, and total protein levels were determined using a bicinchoninic acid assay kit (Pierce, Rockford, IL, USA). Proteins $(15 \mu \mathrm{g})$ were separated on $12 \%$ sodium dodecyl sulfatepolyacrylamide gels and transferred onto polyvinylidene fluoride membranes in a wet transfer device $(30 \mathrm{~V}, 1 \mathrm{~h})$. Membranes were preincubated in 5\% bovine serum albumin for $2 \mathrm{~h}$ and then incubated with the following primary antibodies overnight at $4^{\circ} \mathrm{C}$ : rabbit anti-GFAP, anti-NLRP3, anti-caspase-1, anti-interleukin- (IL-) 18, anti-Bax, anti$\mathrm{Bcl} 2$, and anti-p-ERK (all at 1:1000 dilution and from ProteinTech Group, Rosemont, IL, USA). After incubation with horseradish peroxidase-conjugated anti-rabbit secondary antibodies, as appropriate, for $1 \mathrm{~h}$ ( $1: 5000$; KPL, Gaithersburg, MD, USA), the membranes were reacted with an enhanced chemiluminescence reagent (New England Lab, Woburn, MA, USA). Finally, specific protein bands were visualized by using the ImageQuant LAS 4000 imaging system (GE Healthcare Life Sciences, Issaquah, WA, USA). Intensities of the protein bands were quantified using ImageJ software (NIH; https://imagej.nih.gov/ij/).

2.5. RNA Isolation and Real-Time RT-PCR. Total DNA was isolated from the cerebral cortex and hippocampus tissues. Then, according to the manufacturer's protocol, TransScript One-Step gDNA Removal and cDNA Synthesis SuperMix kits (TransGen, Beijing, China) were used to prepare complementary DNA. The GFAP gene expressions were analyzed in terms of quantity by running RT-PCR by the use of fluorescent SYBR Green technology. Relative expression of the GFAP gene was normalized to $\beta$-actin. The primer was as follows: $\beta$-actin: forward primer: 5 -CGATGCCCTGAGGG TCTTT- $3^{\prime}$ and reverse primer: $5^{\prime}$-TGGATGCCACAGGA TTCCAT-3', and GFAP: forward primer: $5^{\prime}$-TTGCTG GAGGGCGAAGAAA-3' and reverse primer: $5^{\prime}$-AGGGAG AGCTGGCAGG-3'. 

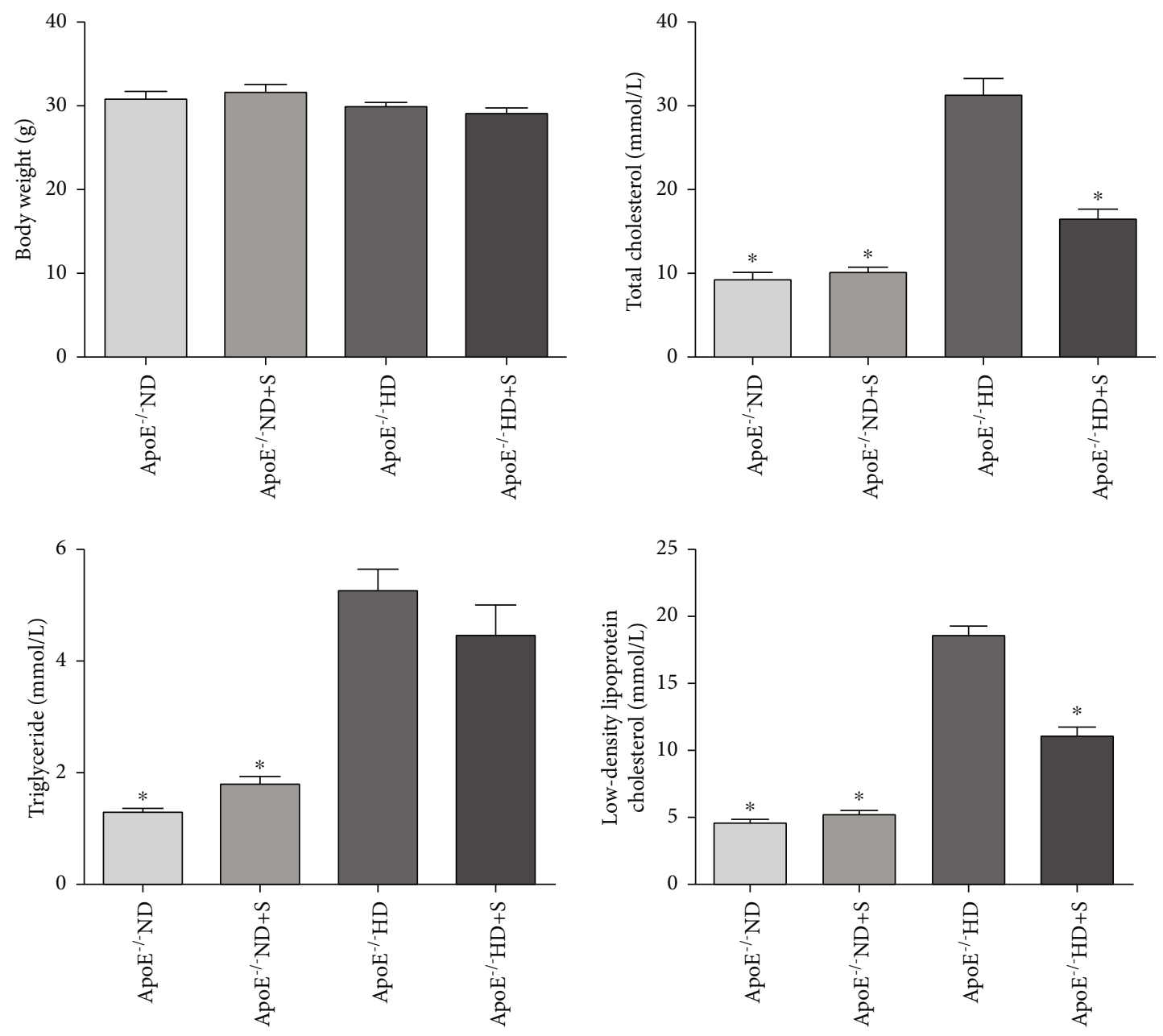

FIGURE 1: Body weights and total cholesterol, triglyceride, and low-density lipoprotein levels in mice after 12 weeks of feeding with different diets. Data are presented as the mean \pm standard error of the mean. $n=7$ per group. ApoE ${ }^{-/-}$: apolipoprotein E-deficient; HD: high-fat diet; ND: normal diet; S: swimming.

2.6. Statistical Analyses. Data are presented as the mean \pm standard error of mean and were analyzed using SPSS software version 23.0 (IBM, Chicago, IL, USA). Intergroup differences were determined by an analysis of variance and Tukey's post hoc test. $P<0.05$ was regarded as significant.

\section{Results}

3.1. Metabolic Characterization. The metabolic characteristics of the animals are shown in Figure 1. Body weights did not differ significantly among the four groups. The levels of LDL-c, TC, and TGs were significantly increased $(P<0.05)$ in the $\mathrm{ApoE}^{-/-} \mathrm{HD}$ groups compared with the ND and ND $+S$ groups. In addition, the levels of LDL-c and TC were decreased in the $\mathrm{HD}+\mathrm{S}$ group compared to the HD group. However, TG levels did not show this decrease. These results suggest that exercise was effective in reducing TC and LDL levels in HD mice.

3.2. Exercise Inhibits the Increased GFAP Expression Caused by Hyperlipidemia in the Cerebral Cortical Layer and Hippocampal Area. To investigate the pathological changes in hyperlipidemia-induced neuroinflammation, we performed GFAP immunohistochemical staining (Figure 2) of different brain tissues (cerebral cortex, hippocampal CA1 area, hippocampal CA3 area, and dentate gyrus). The results showed that GFAP-positive cells were increased in the $\mathrm{ApoE}^{-}$ ${ }^{1-} \mathrm{HD}$ group compared with the ND and ND+S groups. Interestingly, swimming exercise ( $\mathrm{HD}+\mathrm{S}$ group) decreased the number of GFAP-positive cells. In addition, in the ND group, GFAP-positive glial cells had small nuclei and short protrusions, while in the HD group, glial cells were activated, the nuclei were enlarged and rounded, and the protrusions were elongated. After exercise, glial cell activity decreased, the nuclei became smaller, and the protrusions shrank compared to the HD group. Furthermore, real-time PCR and western blot analysis of GFAP showed that the expression of GFAP was inhibited by exercise (Figure 3 ).

\subsection{Exercise Inhibits Hyperlipidemia-Induced}

Neuroinflammation. To evaluate the involvement of proinflammatory cytokines and cell death factors in neuronal tissues from each of the four groups, NLRP3, IL-18, and caspase-1 protein expression were measured using western 


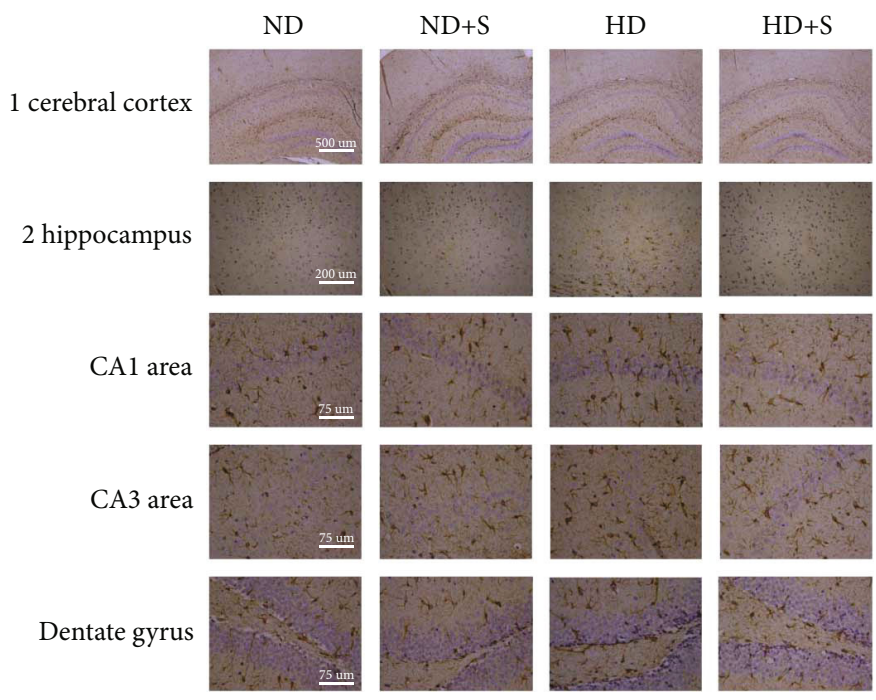

FIGURE 2: Immunohistochemistry showing glial fibrillary acidic protein (GFAP) expression in the (1) cerebral cortex and (2) hippocampus CA1 area, hippocampus CA3 area, and dentate gyrus. ApoE ${ }^{-/}$: apolipoprotein E-deficient; HD: high-fat diet; ND: normal diet; S: swimming.

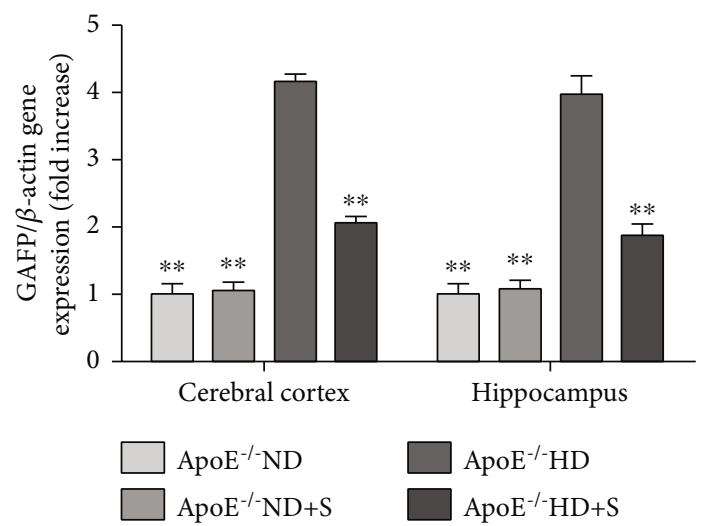

(a)

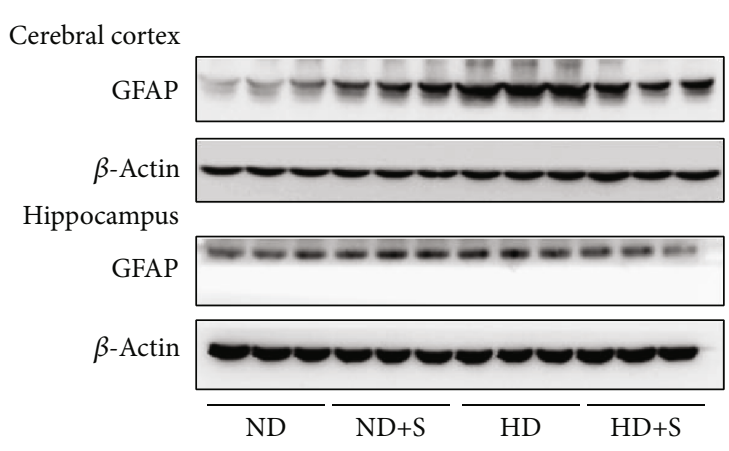

(b)

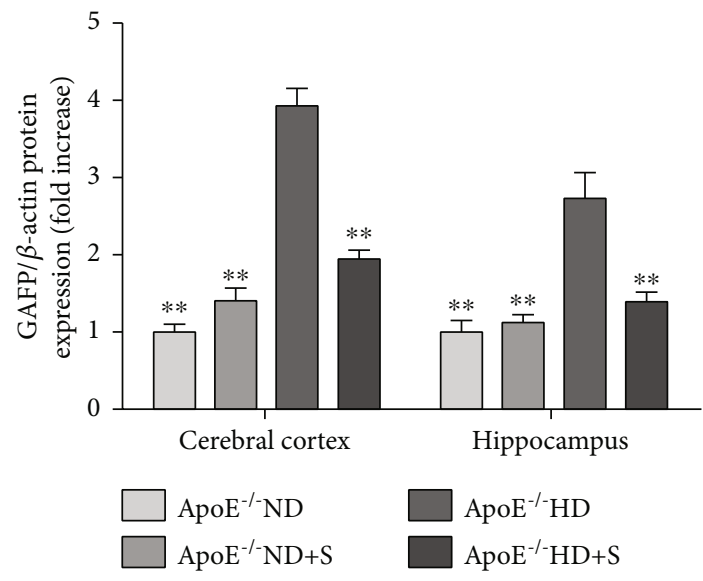

(c)

FIGURE 3: (a) Relative mRNA expression of glial fibrillary acidic protein (GFAP) in the cerebral cortex and hippocampus area. (b) Immunoblotting for GFAP protein expression in the cerebral cortex and hippocampus area. Bar graphs showing quantification of GFAP protein expression. Data are presented as the mean \pm standard error of mean; $n=3$ per group. ${ }^{* *} P<0.01$ vs. the ApoE ${ }^{-/-} \mathrm{HD}_{\text {group. }}{ }^{*} P<$ 0.05 vs. the $\mathrm{ApoE}^{-/-} \mathrm{HD}$ group. ApoE ${ }^{-/-}$: apolipoprotein E-deficient; HD: high-fat diet; ND: normal diet; S: swimming. 

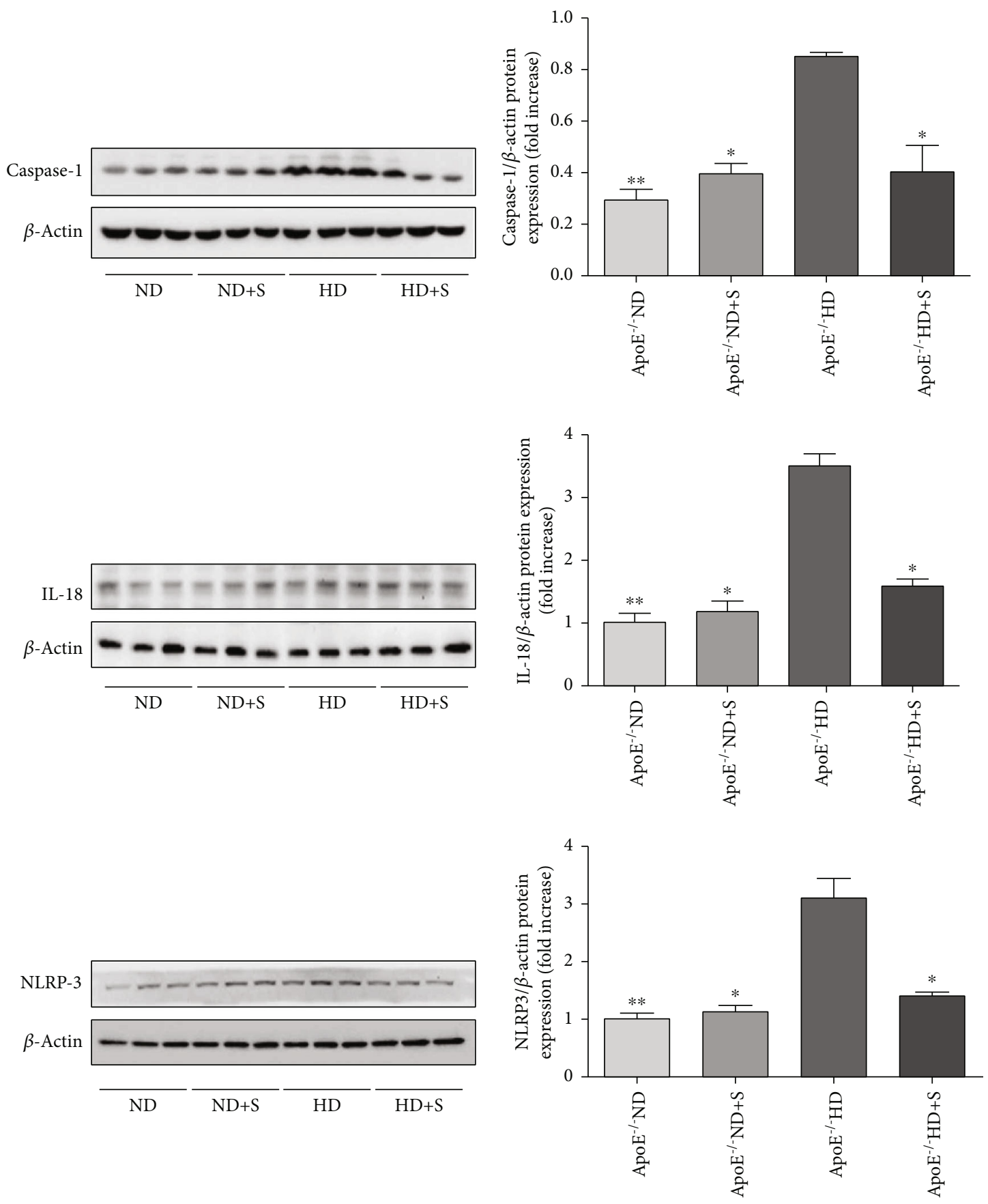

(a)

(b)

FIGURE 4: (a) Immunoblotting for NLR family pyrin domain-containing 3 (NLRP3), interleukin- (IL-) 18, and caspase-1 protein expression in neuronal tissues. (b) Bar graphs showing quantification of NLRP3, IL-18, and caspase-1 protein expression. Data are presented as the mean \pm standard error of mean; $n=3$ per group. ${ }^{* *} P<0.01$ vs. the $\mathrm{ApoE}^{-/-} \mathrm{HD}$ group. ${ }^{*} P<0.05$ vs. the ApoE ${ }^{-/-} \mathrm{HD}$ group. ApoE ${ }^{-/-}$: apolipoprotein E-deficient; HD: high-fat diet; ND: normal diet; S: swimming.

blotting (Figure 4). All three proteins were increased in the $\mathrm{HD}$ group compared to the ND group. However, these increases were attenuated in the $\mathrm{HD}+\mathrm{S}$ group.

3.4. Exercise Inhibits Apoptosis Induced by Hyperlipidemia. To evaluate apoptosis in neuronal tissues of the four experimental groups, Bax and Bcl-2 protein expression was measured using western blotting (Figure 5). The Bax protein level was higher in the HD group than in the ND group. This increase was attenuated in the $\mathrm{HD}+\mathrm{S}$ group. Interestingly, the expression of $\mathrm{Bcl}-2$ showed the opposite trend. Compared to the ND group, Bcl-2 expression was decreased, and this decrease was attenuated by exercise in the $\mathrm{HD}+\mathrm{S}$ group.

3.5. p-ERK Signaling Pathway. Western blotting analysis of p-ERK was used to investigate neuronal damage caused by 

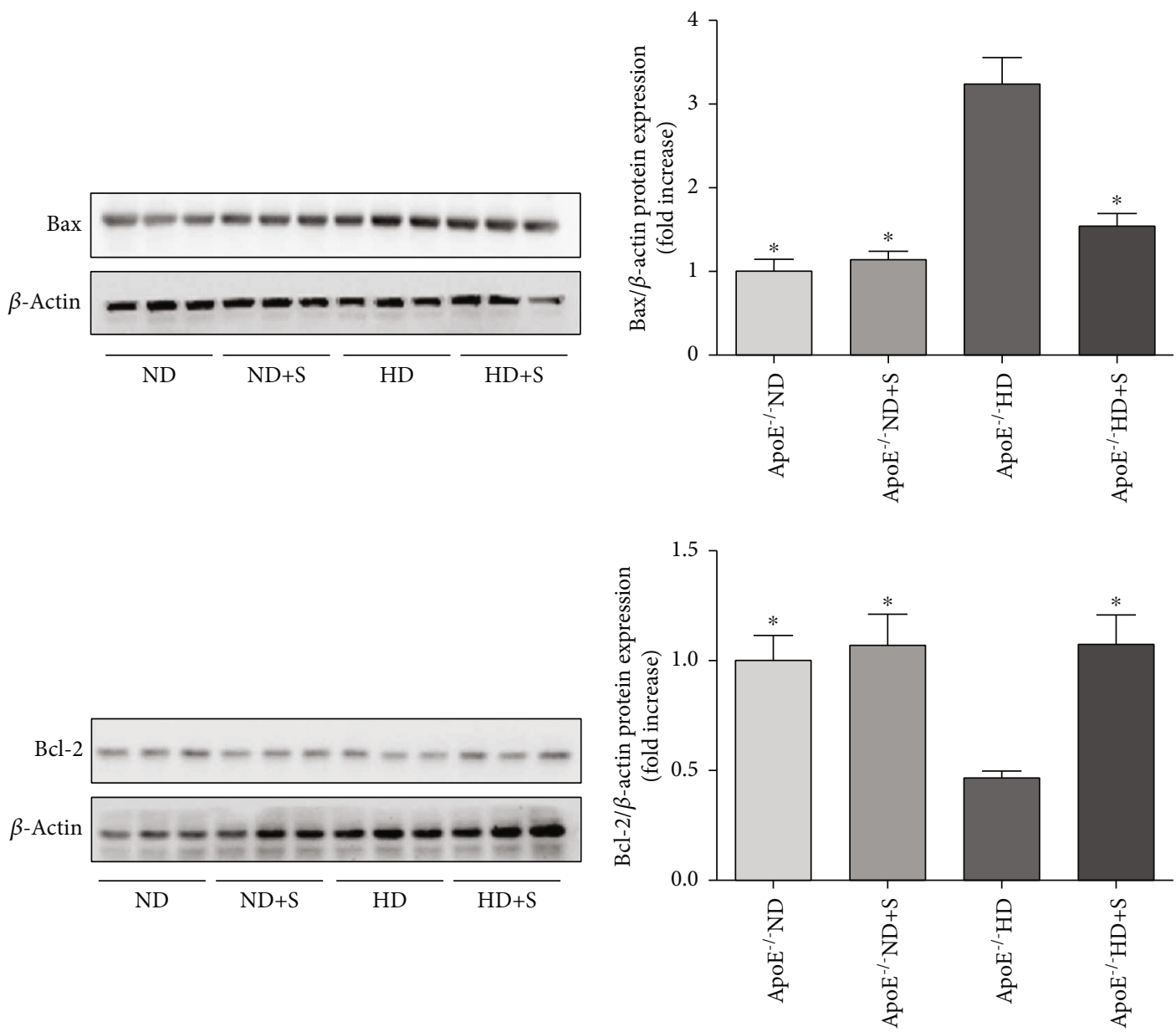

(a)

(b)

FIGURE 5: (a) Immunoblotting for Bax and Bcl-2 protein expression in neuronal tissues. (b) Bar graphs showing quantification of Bax and Bcl-

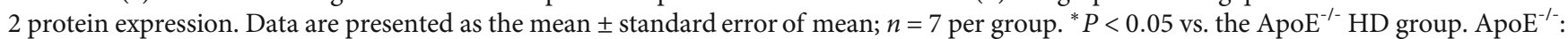
apolipoprotein E-deficient; HD: high-fat diet; ND: normal diet; S: swimming.

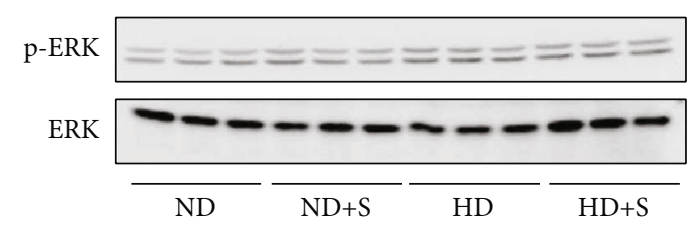

(a)

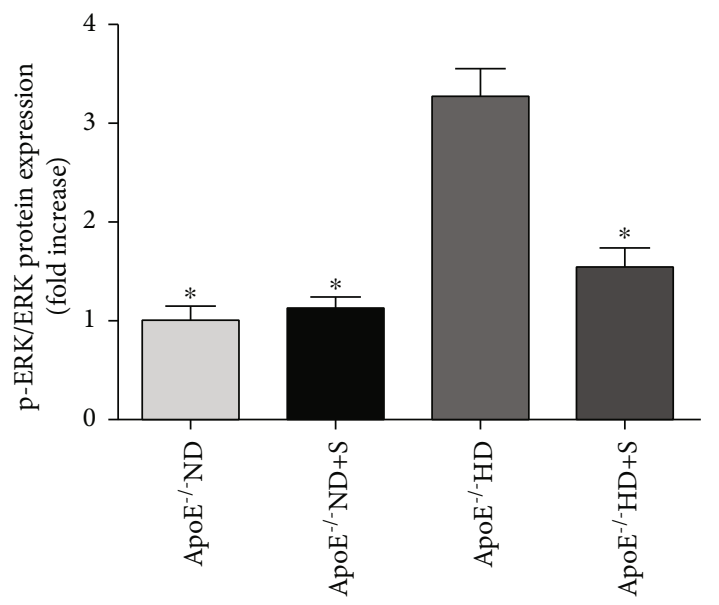

(b)

FIGURE 6: (a) Immunoblotting for phosphorylated extracellular signal-regulated kinase (p-ERK) protein expression in neuronal tissues. (b) Bar graph showing quantification of p-ERK protein expression. Data are presented as the mean \pm standard error of mean; $n=3$ per group. ${ }^{*} P<0.05$ vs. the $\mathrm{ApoE}^{-/-} \mathrm{HD}$ group. ApoE ${ }^{-/-}$: apolipoprotein E-deficient; HD: high-fat diet; ND: normal diet; S: swimming. 
hyperlipidemia (Figure 6). p-ERK protein expression in neuronal tissues was lower in $\mathrm{ApoE}^{-1-} \mathrm{HD}+\mathrm{S}$ mice than in ApoE $^{-/-} \mathrm{HD}$ mice.

\section{Discussion}

In this study, we explored the protective effects of exercise using a model of hyperlipidemia-induced neuronal injury in $\mathrm{ApoE}^{-/-}$mice. The results demonstrated that exercise had a protective effect on this injury by affecting proinflammatory cytokine levels, apoptosis, GFAP expression, and the p-ERK pathway.

No significant variation was observed in body weights among the four groups of mice. However, compared with ApoE $^{-/-}$ND group mice, higher LDL-c and TC levels were observed in $\mathrm{ApoE}^{-/-} \mathrm{HD}$ group mice. This result indicated that the hyperlipidemia mouse model was established successfully by a high-fat diet. A review article noted that exercise has an effect on the serum levels of cholesterol and lipids [18]. Interestingly, LDL-c and TC levels were significantly lower in the $\mathrm{ApoE}^{-/-} \mathrm{HD}+\mathrm{S}$ group than in the $\mathrm{ApoE}^{-}$

/- HD group, suggesting that exercise had a protective effect against lipid deposition caused by a HD. However, the effect of exercise on hyperlipidemia-induced neuronal injury remained unclear.

Immunohistochemistry, real-time PCR, and immunoblotting were performed to evaluate GFAP levels for understanding the mechanism of neuronal damage caused by hyperlipidemia. Astrocytes play a significant role in maintaining the physiological functions of the blood-brain barrier and in regulating the metabolism of lipids in the brain [19]. GFAP, an intermediate filament protein that is primarily expressed in astrocytes, is a key marker of mature astrocytes [20]. Many studies have shown that overexpression of GFAP is associated with neuronal damage. Stolmeier et al. reported that increases in GFAP and Ibal antagonized hippocampal function [21]. In the current study, the expression of GFAP was increased in the HD group. This showed that hyperlipidemia activated glial cells and caused neuronal damage. Importantly, this effect was suppressed by exercise.

Hyperlipidemia-induced inflammation plays a crucial role in the development of cardiac damage, ischemic stroke, and brain injury [22]. Neuroinflammation has been identified as a causative factor of multiple neurological diseases $[23,24]$. The nucleotide-binding oligomerization domain-, leucine-rich repeat-, and pyrin domain-containing 3 (NLRP3) inflammasome is abundantly expressed in the central nervous system and is the most studied inflammasome in this system $[25,26]$. Activating the NLRP3 inflammasome leads to the activation of caspase-1. Subsequently, activated caspase- 1 causes the production of IL- $1 \beta$ and IL-18, as well as proinflammatory cytokines, and mediates rapid cell death. IL- $1 \beta$ and IL-18 drive inflammatory responses through a variety of downstream signaling pathways that result in neuronal injury $[12,27,28]$. Results of the current study showed that NLRP3, caspase-1, and IL-18 levels were increased in the HD group. In this study, swimming, as a treatment, reduced the expression of NLRP3, caspase-1, and IL-18, thereby indi- cating that exercise can act as a protective agent against hyperlipidemia-induced neuronal injury.

Apoptosis plays an important role in various neuronal injuries [29-31]. Kim et al. found that berberine treatment inhibited brain inflammation in poloxamer 407-treated hyperlipidemic rats by inhibiting apoptosis [32]. The key regulators of apoptosis are members of the Bcl-2 family of proteins; this protein family contains a variety of proapoptotic (such as Bax and Bak) and antiapoptotic (such as Bcl-2, $\mathrm{Bcl}-\mathrm{xL}$, and $\mathrm{Bcl}-\mathrm{w})$ proteins $[33,34]$. In the current study, compared to the ND group, Bax protein levels were increased and $\mathrm{Bcl}-2$ protein levels decreased in the HD group. It is worth noting that exercise inhibited the expression of Bax and enhanced the expression of Bcl-2, suggesting that apoptosis was inhibited.

The mitogen-activated protein kinase/ERK pathway participates in every stage of cell growth and development, including cell proliferation, differentiation, migration, senescence, and apoptosis [35]. In white matter-lesioned rats, activation of the mitogen-activated protein kinase/ERK pathway promotes neuronal apoptosis, thereby worsening the condition [36]. In the current study, we found that p-ERK expression was significantly increased in the HD group. This showed that hyperlipidemia caused neuronal injury by activating the p-ERK signaling pathway, and exercise can inhibit this effect.

\section{Conclusions}

In conclusion, the results of the present study showed that exercise treatment had a protective effect on hyperlipidemiainduced neuronal injury in $\mathrm{ApoE}^{-/-}$mice. Exercise reduced the increases in serum LDL-c and TC and protected against neuronal damage by inhibiting GFAP expression, inflammation, apoptosis, and activation of the p-ERK signaling pathway. The findings of this study could be beneficial in developing novel strategies for the prevention and treatment of neuronal injury.

\section{Data Availability}

All datasets are available from the corresponding author upon reasonable request.

\section{Conflicts of Interest}

The authors declare that there is no conflict of interest regarding the publication of this paper.

\section{References}

[1] J. Banefelt, S. Hallberg, K. M. Fox et al., "Work productivity loss and indirect costs associated with new cardiovascular events in high-risk patients with hyperlipidemia: estimates from population-based register data in Sweden," The European Journal of Health Economics, vol. 17, no. 9, pp. 11171124, 2016.

[2] M. Ding, D. Si, W. Zhang, Z. Feng, M. He, and P. Yang, "Red yeast rice repairs kidney damage and reduces inflammatory transcription factors in rat models of hyperlipidemia," 
Experimental and Therapeutic Medicine, vol. 8, no. 6, pp. 1737-1744, 2014.

[3] Q. Wang, J. Yuan, Z. Yu et al., "FGF21 attenuates high-fat dietinduced cognitive impairment via metabolic regulation and anti-inflammation of obese mice," Molecular Neurobiology, vol. 55, no. 6, pp. 4702-4717, 2018.

[4] A. M. Vincent, L. M. Hinder, R. Pop-Busui, and E. L. Feldman, "Hyperlipidemia: a new therapeutic target for diabetic neuropathy," Journal of the Peripheral Nervous System, vol. 14, no. 4, pp. 257-267, 2009.

[5] N. Pierrot and J. N. Octave, "Cholesterol, neuronal activity and Alzheimer disease,” Medecine Sciences, vol. 30, no. 3, pp. 244246, 2014.

[6] N. E. Shepardson, G. M. Shankar, and D. J. Selkoe, "Cholesterol level and statin use in Alzheimer disease: I. Review of epidemiological and preclinical studies," Archives of Neurology, vol. 68, no. 10, pp. 1239-1244, 2011.

[7] J. Chen, L. G. Costa, and M. Guizzetti, “Assessment of cholesterol homeostasis in astrocytes and neurons," Methods in Molecular Biology (Clifton, NJ), vol. 758, pp. 403-414, 2011.

[8] K. Y. Kim, M. V. Stevens, M. H. Akter et al., "Parkin is a lipidresponsive regulator of fat uptake in mice and mutant human cells," The Journal of clinical investigation., vol. 121, no. 9, pp. 3701-3712, 2011.

[9] R. Paul, A. Choudhury, S. Kumar, A. Giri, R. Sandhir, and A. Borah, "Cholesterol contributes to dopamine-neuronal loss in MPTP mouse model of Parkinson's disease: involvement of mitochondrial dysfunctions and oxidative stress," PloS One, vol. 12, no. 2, article e0171285, 2017.

[10] K. Deckers, M. P. van Boxtel, O. J. Schiepers et al., "Target risk factors for dementia prevention: a systematic review and Delphi consensus study on the evidence from observational studies," International Journal of Geriatric Psychiatry, vol. 30, no. 3, pp. 234-246, 2015.

[11] A. Jayaraman, D. Lent-Schochet, and C. J. Pike, "Diet-induced obesity and low testosterone increase neuroinflammation and impair neural function," Journal of Neuroinflammation, vol. 11, no. 1, p. 162, 2014.

[12] S. L. Fink, T. Bergsbaken, and B. T. Cookson, "Anthrax lethal toxin and Salmonella elicit the common cell death pathway of caspase-1-dependent pyroptosis via distinct mechanisms," Proceedings of the National Academy of Sciences of the United States of America, vol. 105, no. 11, pp. 4312-4317, 2008.

[13] H. Wang, N. K. Liu, Y. P. Zhang et al., "Treadmill training induced lumbar motoneuron dendritic plasticity and behavior recovery in adult rats after a thoracic contusive spinal cord injury," Experimental Neurology, vol. 271, pp. 368-378, 2015.

[14] J. C. Petruska, R. M. Ichiyama, D. L. Jindrich et al., "Changes in motoneuron properties and synaptic inputs related to step training after spinal cord transection in rats," The Journal of Neuroscience, vol. 27, no. 16, pp. 4460-4471, 2007.

[15] M. A. Shibata, E. Shibata, K. Maemura, Y. Kondo, and M. Harada-Shiba, "Pathological and molecular analyses of atherosclerotic lesions in ApoE-knockout mice," Medical Molecular Morphology, vol. 50, no. 3, pp. 130-144, 2017.

[16] J. A. Piedrahita, S. H. Zhang, J. R. Hagaman, P. M. Oliver, and N. Maeda, "Generation of mice carrying a mutant apolipoprotein $\mathrm{E}$ gene inactivated by gene targeting in embryonic stem cells," Proceedings of the National Academy of Sciences of the United States of America, vol. 89, no. 10, pp. 44714475, 1992.
[17] C. Sastre, A. Rubio-Navarro, I. Buendía et al., "Hyperlipidemia-associated renal damage decreases Klotho expression in kidneys from ApoE knockout mice," PloS One, vol. 8, no. 12, article e83713, 2013

[18] S. Mann, C. Beedie, and A. Jimenez, "Differential effects of aerobic exercise, resistance training and combined exercise modalities on cholesterol and the lipid profile: review, synthesis and recommendations," Sports Medicine (Auckland, NZ), vol. 44, no. 2, pp. 211-221, 2014.

[19] M. V. Sofroniew and H. V. Vinters, "Astrocytes: biology and pathology," Acta Neuropathologica, vol. 119, no. 1, pp. 7-35, 2010.

[20] L. F. Eng and R. S. Ghirnikar, "GFAP and astrogliosis," Brain Pathology (Zurich, Switzerland), vol. 4, no. 3, pp. 229-237, 1994.

[21] D. Stolmeier, R. Thangavel, P. Anantharam, M. M. Khan, D. Kempuraj, and A. Zaheer, "Glia maturation factor expression in hippocampus of human Alzheimer's disease," Neurochemical Research, vol. 38, no. 8, pp. 1580-1589, 2013.

[22] R. Jin, G. Yang, and G. Li, "Inflammatory mechanisms in ischemic stroke: role of inflammatory cells," Journal of Leukocyte Biology, vol. 87, no. 5, pp. 779-789, 2010.

[23] D. Y. Choi, M. Liu, R. L. Hunter et al., "Striatal neuroinflammation promotes Parkinsonism in rats," PloS One, vol. 4, no. 5, article e5482, 2009.

[24] H. Abo-ouf, A. W. Hooper, E. J. White, H. J. Janse van Rensburg, B. L. Trigatti, and S. A. Igdoura, "Deletion of tumor necrosis factor- $\alpha$ ameliorates neurodegeneration in Sandhoff disease mice," Human Molecular Genetics, vol. 22, no. 19, pp. 3960-3975, 2013.

[25] J. S. Wallisch, D. W. Simon, H. Bayır, M. J. Bell, P. M. Kochanek, and R. S. B. Clark, "Cerebrospinal fluid NLRP3 is increased after severe traumatic brain injury in infants and children," Neurocritical Care, vol. 27, no. 1, pp. 4450, 2017.

[26] A. Halle, V. Hornung, G. C. Petzold et al., "The NALP3 inflammasome is involved in the innate immune response to amyloid- $\beta$," Nature Immunology, vol. 9, no. 8, pp. 857-865, 2008.

[27] L. Song, L. Pei, S. Yao, Y. Wu, and Y. Shang, "NLRP3 inflammasome in neurological diseases, from functions to therapies," Frontiers in Cellular Neuroscience, vol. 11, p. 63, 2017.

[28] K. Mortezaee, N. Khanlarkhani, C. Beyer, and A. Zendedel, "Inflammasome: its role in traumatic brain and spinal cord injury," Journal of Cellular Physiology, vol. 233, no. 7, pp. 5160-5169, 2018.

[29] E. Pajarillo, A. Rizor, D.-S. Son, M. Aschner, and E. Lee, "The transcription factor REST up-regulates tyrosine hydroxylase and antiapoptotic genes and protects dopaminergic neurons against manganese toxicity," Journal of Biological Chemistry., vol. 295, no. 10, pp. 3040-3054, 2020.

[30] Q. S. Liu, R. Deng, S. Li et al., "Ellagic acid protects against neuron damage in ischemic stroke through regulating the ratio of Bcl-2/Bax expression," Applied Physiology, Nutrition, and Metabolism, vol. 42, no. 8, pp. 855-860, 2017.

[31] A. Nakamura, V. Swahari, C. Plestant et al., "Bcl-xL is essential for the survival and function of differentiated neurons in the cortex that control complex behaviors," The Journal of Neuroscience, vol. 36, no. 20, pp. 5448-5461, 2016.

[32] M. Kim, T. W. Kim, C. J. Kim et al., "Berberine ameliorates brain inflammation in poloxamer 407 -induced hyperlipidemic 
rats," International Neurourology Journal, vol. 23, Supplement 2, pp. S102-S110, 2019.

[33] R. J. Youle and A. Strasser, "The BCL-2 protein family: opposing activities that mediate cell death," Nature Reviews Molecular Cell Biology, vol. 9, no. 1, pp. 47-59, 2008.

[34] J. E. Chipuk, T. Moldoveanu, F. Llambi, M. J. Parsons, and D. R. Green, "The BCL-2 family reunion," Molecular Cell, vol. 37, no. 3, pp. 299-310, 2010.

[35] J. Y. Lim, S. I. Park, J. H. Oh et al., "Brain-derived neurotrophic factor stimulates the neural differentiation of human umbilical cord blood-derived mesenchymal stem cells and survival of differentiated cells through MAPK/ERK and PI3K/Akt-dependent signaling pathways," Journal of Neuroscience Research, vol. 86, no. 10, pp. 2168-2178, 2008.

[36] Y. Guo, C. Liu, J. Zhang et al., "A relationship between MAPK/ERK pathway expression and neuronal apoptosis in rats with white matter lesions," European Review for Medical and Pharmacological Sciences, vol. 24, no. 8, pp. 4412-4419, 2020. 